\title{
Compliance, Counseling and Satisfaction with Oral Contraceptives: A Prospective Evaluation
}

\author{
By Michael J. Rosenberg, Michael S. Waugh and Michael S. Burnhill
}

Context: Oral contraceptive users' risk of accidental pregnancy may be higher than it should be, because of inconsistent pill-taking. However, few reliable data are available on pill users' everyday experiences with their method, especially characteristics that may affect consistency of use.

Methods: Two months after initiating or resuming oral contraceptive use, a nationwide sample of 943 women completed questionnaires examining their compliance with instructions for proper use, the quality of their interactions with their provider, their satisfaction with the method, and the frequency and costs of visits or calls to their providers because of pill-related side effects. Regression analyses were used to determine the factors associated with compliance difficulties and method dissatisfaction.

Results: In all, $47 \%$ of users missed one or more pills per cycle, and $22 \%$ missed two or more. Women who lacked an established pill-taking routine, who did not read and understand all of the informational material accompanying the pill package, or who experienced spotting or heavy bleeding had increased odds of missing two or more pills per cycle. Method satisfaction was most likely among women who were aware of the pill's noncontraceptive benefits, were satisfied with their relationship with their provider, had used the pill in the past and experienced few side effects. Some $22 \%$ of users called their provider at least once about pill-related side effects, and 9\% made at least one visit for this reason; these women spent \$25 and \$62, respectively, to treat side effects.

Conclusions: Improving pill use is a shared responsibility of the provider, the patient and, to a lesser degree, pill manufacturers. Awareness of potential difficulties such as inadequate counseling is a key step in helping women use oral contraceptives effectively.

Family Planning Perspectives, 30(2):89-92 \& 104, 1998

$\mathrm{O}$ ral contraceptives are the most popular form of reversible contraception in the United States, used by more than 18 million women. ${ }^{1}$ During the more than 35 years that the pill has been available, considerable resources have been applied to investigating its health effects. ${ }^{2}$ As a consequence of this research and resulting product refinements, oral contraceptives are one of the safest drugs marketed; their safety is particularly striking in comparison with the health risks posed by the pregnancies that pill use prevents. ${ }^{3}$

In contrast, resources spent investigating the dynamics of oral contraceptive use-including women's everyday experience with counseling, compliance with the pill regimen and with instructions about what to do if pills are missed, method satisfaction and discontinuationhave been limited. The importance of these factors is indicated by several observations.

First, only $50-75 \%$ of women who start taking pills and do not want to become pregnant still are using them after one year; this continuation rate is lower than those for other methods with similar contraceptive efficacy if used perfectly. Second, com- pliance difficulties may be substantially more common than generally appreciated. For example, in a recent study, one-third of women using oral contraceptives reported that they miss one or more pills per cycle. ${ }^{5}$ Third, whereas only $0.1 \%$ of pill users would be expected to become pregnant within one year of perfect use, typical pregnancy rates range from $3 \%$ to $8 \%{ }^{6}$

Furthermore, these rates underestimate the problem, since difficulties with oral contraceptives often lead to discontinuation and, in many cases, subsequent unintended pregnancy. ${ }^{7}$ Together, unintended pregnancies following pill discontinuation and compliance difficulties are estimated to account for $20 \%$ of the 3.5 million annual unintended pregnancies in the United States, with associated costs of $\$ 2.6$ billion. ${ }^{8}$

To improve our understanding of women's experiences with oral contraceptives in everyday use, we conducted a nationwide, prospective cohort study of women initiating contraceptive use or switching to a new method. This article focuses on pill users' compliance with instructions for using the method, their satisfaction with the counseling they received about oral contraceptives and with the method itself, and the frequency and costs of pill users' return visits or calls to providers resulting from method-related side effects.

\section{Methods \\ Study Design}

The Organon Contraceptive Use Study included women who obtained contraceptives from physicians in private practice, two Planned Parenthood affiliates and a health maintenance organization between March 1995 and May 1996. An invitation to enroll participants was mailed to the 4,000 physicians in private practice who provide the largest number of pill prescriptions annually (according to data provided by the marketing firm IMS America), and to 10 Planned Parenthood affiliates and 10 health maintenance organizations. Responses were received from 878 physicians throughout the United States, of whom 271 agreed to enroll participants.

Clinicians were instructed to alternate prescribing Desogen (a combined pill manufactured by Organon) and any other brand of pill to their patients who were either beginning oral contraceptive use or switching brands. At enrollment in the study, women received a questionnaire from their provider, which they returned by mail to the researchers. Two months later, a follow-up questionnaire was mailed directly to the participant, accompanied by a nominal cash incentive. If a response was not received within 14 days of the first mailing, a second copy of the questionnaire was mailed with a reminder note, without a cash incentive.

Women who did not respond to the second mailing within 14 days were considered nonrespondents. We attempted to contact these women by telephone within an additional 14 days. If women could not be contacted by phone after two attempts or still failed to return the ques-

Michael J. Rosenberg is president of the research organization Health Decisions, Chapel Hill, NC, and clinical professor, Departments of Obstetrics-Gynecology and Epidemiology, University of North Carolina, Chapel Hill; Michael S. Waugh is senior project director, Health Decisions; and Michael S. Burnhill is medical director, Planned Parenthood Federation of America, New York. This research was funded in part by an unrestricted grant from Organon. 
Table 1. Percentage distribution or means of characteristics of respondents and women lost to follow-up, Organon Contraceptive Use Study, 1995-1996

\begin{tabular}{llll}
\hline Characteristic & $\begin{array}{l}\text { Replied } \\
\text { to initial } \\
\text { mailing }\end{array}$ & $\begin{array}{l}\text { Replied } \\
\text { after } \\
\text { phone } \\
\text { follow-up } \\
(\mathrm{N}=303)\end{array}$ & $\begin{array}{l}\text { Lost to } \\
\text { follow-up }\end{array}$ \\
& $(\mathrm{N}=5653)$ \\
\hline
\end{tabular}

\section{\% DISTRIBUTION}

\section{Education}

$<$ high school

Completed

high school 21

Some college $\quad 37$

Completed college 24

Postgraduate 9

No response

1

Annual household income

\begin{tabular}{|c|c|c|c|}
\hline$<\$ 15,000$ & 25 & 28 & 29 \\
\hline$\$ 15,000-24,999$ & 17 & 18 & 18 \\
\hline$\$ 25,000-34,999$ & 13 & 13 & 13 \\
\hline$\$ 35,000-54,999$ & 16 & 15 & 13 \\
\hline$\geq \$ 55,000$ & 16 & 15 & 11 \\
\hline \multicolumn{4}{|l|}{ Don't know/ } \\
\hline no response & 13 & 12 & 15 \\
\hline \multicolumn{4}{|l|}{ Marital status } \\
\hline Never-married & 65 & 62 & 64 \\
\hline Married & 25 & 30 & 26 \\
\hline \multicolumn{4}{|c|}{ Separated/divorced/ } \\
\hline widowed & 10 & 8 & 10 \\
\hline \multicolumn{4}{|c|}{ Method initiated at enrollment } \\
\hline Pill & 95 & 95 & 95 \\
\hline Male condom & 0 & 0 & 1 \\
\hline Injectable & 2 & 3 & 2 \\
\hline Implant & 0 & 0 & 0 \\
\hline Other & 3 & 2 & 2 \\
\hline \multicolumn{4}{|l|}{ Past pill use } \\
\hline Never & 30 & 30 & 31 \\
\hline Before previous & & & \\
\hline month & 39 & 43 & 46 \\
\hline Previous month & 31 & 27 & 23 \\
\hline \multicolumn{4}{|l|}{ Parity } \\
\hline 0 & 48 & 46 & 40 \\
\hline$\geq 1$ & 52 & 54 & 60 \\
\hline Total & 100 & 100 & 100 \\
\hline \multicolumn{4}{|l|}{ MEAN } \\
\hline Age at enrollment & 26 & 25 & 26 \\
\hline Age at first sex & 16 & 16 & 16 \\
\hline Lifetime no. of & 6 & 6 & 6 \\
\hline
\end{tabular}

Note: Respondents are women who completed a questionnaire two months after enrolling in the study.

tionnaire, they were classified as lost to follow-up. In all, 1,555 women enrolled in the study; of these, 992 (64\%) returned a twomonth questionnaire.

\section{Outcome Measures and Analysis}

On the basis of the distribution of responses, we defined women who missed two or more pills per cycle as having com-

\footnotetext{
*We used missed pills as a surrogate measure for compliance difficulties in general, which include other behaviors (such as failure to use a backup method as indicated and method discontinuation while still at risk for unintended pregnancy) and other situations that may be outside the user's control.
}

pliance difficulties. ${ }^{*}$ Findings from a previously published literature review on oral contraceptive compliance were used to guide our selection of variables for a comprehensive questionnaire assessing the determinants of compliance. ${ }^{9}$ The review found that three kinds of variables predict patient compliance: user characteristics, health beliefs (i.e., the patient's assessment of costs vs. benefits of a health action, such as following instructions for correct pill use) and characteristics of the patientprovider interaction.

We used logistic regression to identify factors that independently predicted compliance difficulties. Our final selection of variables for the regression analyses followed the "change-in-estimate" method, in which a variable is retained if it changes the risk estimate by $10 \%$ or more. ${ }^{10}$ We included in the analyses variables that resulted in a $10 \%$ or greater change in compliance problems.

To provide a measure of the quality of the patient-provider relationship, at enrollment, participants completed the Patient Reactions Assessment (PRA), a previously validated instrument. ${ }^{11}$ The PRA explores the patient's perception of three areas of this relationship: how well the provider conveyed information, how much the provider seemed to care for and respect the patient, and how easy it was to initiate communication with the provider. Five questions are asked about each area, and responses are recorded on a seven-point scale (very strongly agree to very strongly disagree); thus, a maximum score of 105 is possible.

We also used logistic regression to determine the factors affecting women's satisfaction with the pill. Method satisfaction was measured after two months of oral contraceptive use through scaled responses to five variables: ease of use, effectiveness, troubled by side effects, bothered by cost, overall rating. The choice of responses to "troubled by side effects" and "bothered by cost" ranged from "a great deal" to "not at all," while the choices for the other three variables ranged from "very poor" to "very good." For the regression analysis, these five variables were combined into a single index of satisfaction, with responses dichotomized (poor or good). Logistic regression was used to evaluate the relationship of method satisfaction with the PRA score, the woman's socioeconomic and demographic characteristics, her internal health locus of control, her quality of life and perceived social support, contraceptive cost, and method-related side effects.
The questionnaire asked the women about pill-related side effects that had been bothersome enough to warrant a call or visit to their health care provider. It also asked them to report out-of-pocket expenditures for over-the-counter or prescription drugs, as well as physician visits, to relieve symptoms or side effects associated with oral contraceptive use. Only expenses that were not reimbursed by insurance or other sources are included.

\section{Results \\ Sample Characteristics}

Fifty-six percent of participants were enrolled through private physicians, $42 \%$ through two Planned Parenthood clinics and $2 \%$ through one health maintenance organization. Respondents were young (mean age, 25 years) and primarily nevermarried $(63 \%)$, and had diverse levels of education and income (Table 1). Few differences in characteristics were noted between women who responded to the questionnaire after the initial mailing, those who reponded after telephone follow-up and those who did not respond; the main differences were that nonrespondents were slightly less likely to be nulliparous, were slightly more likely to have used oral contraceptives in the past and were less educated than respondents. Ninety-five percent of participants (943 women) began or switched to new pills at study enrollment.

\section{Compliance}

Almost half (47\%) of pill users reported generally missing one or more pills per cycle, and $22 \%$ missed two or more (i.e., had compliance difficulties). The regression results show that a number of user characteristics and side effects were significantly associated with compliance difficulties. Women who did not have an established routine for their pill-taking were 3.6 times as likely to miss two or more pills per cycle as were women who did have a routine (Table 2). Compared with women who read and understood all written information that came with their pill package, those who read and understood only some of this information were 2.3 times as likely to miss pills, and those who understood little or none of it were 2.8 times as likely to do so.

Women reporting spotting (intermenstrual bleeding not requiring sanitary protection) and heavy or extended periods were 1.6-1.7 times as likely as those not reporting these side effects to miss two or more pills per cycle. The data suggest an association between intermenstrual bleed- 
ing other than spotting (i.e., requiring sanitary protection) and compliance difficulties, but the odds ratio (1.4) was not statistically significant (not shown).

\section{Provider and Method Satisfaction}

The mean PRA score was 88 (range, 25-105; standard deviation, 12.2), indicating that participants were generally satisfied with the care they received from their provider. Women also were largely satisfied with their oral contraceptive: Some $34 \%$ rated it as very good, and another $30 \%$ as good. Satisfaction with the pill tended to be low among women who reported low counseling scores for their providers: Overall, $11 \%$ of women gave their provider low scores and said their satisfaction with the pill was low. However, $25 \%$ reported low PRA scores but moderate levels of satisfaction with the method. The mean PRA score rose steadily from 83 among women reporting very poor satisfaction with the method to 91 among those reporting very good satisfaction.

The relationship between the patientprovider interaction and patient satisfaction with oral contraceptives persisted even after we controlled for other factors that predicted method satisfaction (Table 3). Compared with women who reported the high-

Table 2. Odds ratio (and $95 \%$ confidence interval) from multivariate regression analysis showing the risk of missing two or more pills per cycle, by characteristic

\begin{tabular}{ll}
\hline Characteristic & Odds ratio \\
\hline USE-RELATED & \\
Take pill at same time every day & \\
Yes & 1.0 \\
No & $3.6(2.1-6.3)$ \\
& \\
Amount of package information & \\
read and understood & \\
All & 1.0 \\
Some & $2.3(1.3-4.1)$ \\
Little or none & $2.8(1.5-5.1)$ \\
& \\
Normal pill-taking time & \\
Morning & 1.0 \\
Lunchtime & $0.8(0.4-2.0)$ \\
Afternoon & $1.4(0.6-3.4)$ \\
Evening & $1.4(0.7-2.7)$ \\
Right before bed & $2.6(1.7-4.2)$ \\
None & $9.3(2.1-41.5)$ \\
SIDE EFFECTS & \\
Spotting & \\
No & \\
Yes & 1.0 \\
Heavy or extended periods & $1.7(1.1-2.6)$ \\
No & \\
Yes & 1.0 \\
\hline
\end{tabular}

Note: Regression analysis also controlled for age, pill brand, such side effects as nausea and breast tenderness, where the woman keeps her pills, woman's knowledge of pill-related health benefits, the type of provider prescribing the pill and the woman's perceived level of social support for pill use. These factors did not change the risk estimate by at least $10 \%$, however, and are not included above. est PRA scores, those reporting scores in the bottom two quartiles were 1.6-2.2 times as likely to be dissatisfied with the pill. Levels of dissatisfaction also were elevated among women reporting any side effects; those who were aware of no more than one beneficial effect of pill use; those who did not take the pill at the same time every day; past users who resumed use when entering the study; and women paying the highest prices for oral contraceptives.

\section{Side Effects}

Twenty-two percent of women receiving pill prescriptions called their provider at least once about symptoms related to pill use, and $9 \%$ visited their provider at least once for this reason. For women who called, the most common complaints, reported by $54-55 \%$, were breast tenderness or swelling and worsened mood or more mood changes (Figure 1, page 92); nausea and intermenstrual spotting also were frequent complaints (44-46\%). Among women who returned to their provider because of side effects, $60 \%$ were concerned about weight gain, and 53-54\% complained about changes in mood, breast tenderness or swelling, or nausea. Sixtysix percent of all calls and $75 \%$ of all visits were made by women reporting four or more side effects (not shown).

Costs to women who were bothered enough by side effects to make unscheduled calls or visits were substantial. Excluding patients of the health maintenance organization, women who reported unscheduled calls spent an average of $\$ 25$, and women who reported visits spent an average of $\$ 62$, to treat side effects experienced over the two-month follow-up period.

\section{Discussion}

While the majority of oral contraceptive users in this sample took their pills reliably, nearly half missed one or more pills per cycle, and almost one-quarter missed two or more pills. Additionally, while most women were satisfied with their oral contraceptive, women who were dissatisfied with their interaction with their health care provider were likely also to be dissatisfied with pills as a contraceptive method. These findings, along with those on the predictors of inconsistent use and the frequency and cost of unscheduled calls and visits to providers because of side effects, suggest potential for substantial improvement in how oral contraceptives are used.

The frequency of missed pills reported in this investigation is similar to that found in studies that used pill counts, urine markers and serum progesterone levels to
Table 3. Odds ratio (and 95\% confidence interval) from multivariate regression analysis showing the risk of method dissatisfaction, by characteristic

\begin{tabular}{ll}
\hline Characteristic & Odds ratio \\
\hline No. of side effects & \\
0 & 1.0 \\
$1-3$ & $2.3(1.1-4.7)$ \\
$4-6$ & $6.0(2.9-12.5)$ \\
$\geq 7$ & $9.8(4.0-24.0)$ \\
No. of beneficial effects & \\
$\geq 4$ & \\
3 & 1.0 \\
2 & $1.6(1.0-2.5)$ \\
$0-1$ & $1.6(1.0-2.6)$ \\
& $2.2(1.3-3.6)$
\end{tabular}

Patient Reactions Assessment score* Fourth quartile 1.0 Third quartile

Second quartile 1.0 First quartile

$1.6(1.0-2.5)$ $2.2(1.4-3.5)$

Take pill at same time every day Yes

1.0 No

$1.9(1.3-2.7)$

\section{Past pill use}

Previous month

Never

1.0

Before previous month

$1.3(0.9-1.9)$

$1.8(1.2-2.6)$

Called provider because of side effects No 1.0

Yes

$1.5(1.0-2.2)$

Cost of pills $\dagger$

First quartile

Second quartile

Third quartile

1.0

Fourth quartile

$0.9(0.5-1.6)$

$1.0(0.7-1.5)$

$1.7(1.1-2.7)$

*Scores increase from first through fourth quartiles. + Costs increase from first through fourth quartiles. Note: Regression analysis also of life, locus of control, woman's perceived level of social support for pill use and the type of provider prescribing the pill. These factors did not change the risk estimate by at least $10 \%$, however, and so are not included above.

measure consistency of use; ${ }^{12}$ it is higher than that in a retrospective study based on patients' self-reports. ${ }^{13}$

A recent investigation reporting the frequency of missed pills using electronic monitoring devices as well as diary data provides another perspective. ${ }^{14}$ The proportion of women recording no missed pills in the diary was much higher than the proportion recorded electronically in each of the three months of observation (53-59\% compared with 19-33\%), and the proportion missing at least three pills according to the electronic data was triple that derived from the women's reports (30-51\% vs. $10-14 \%$ ). These results may not be generalizable to all pill users, because they were based on a comparatively small and select sample (103 women recruited from two university student health services and two publicly funded family planning clinics). Nevertheless, they suggest that our findings may underestimate the true frequency of missed pills. 


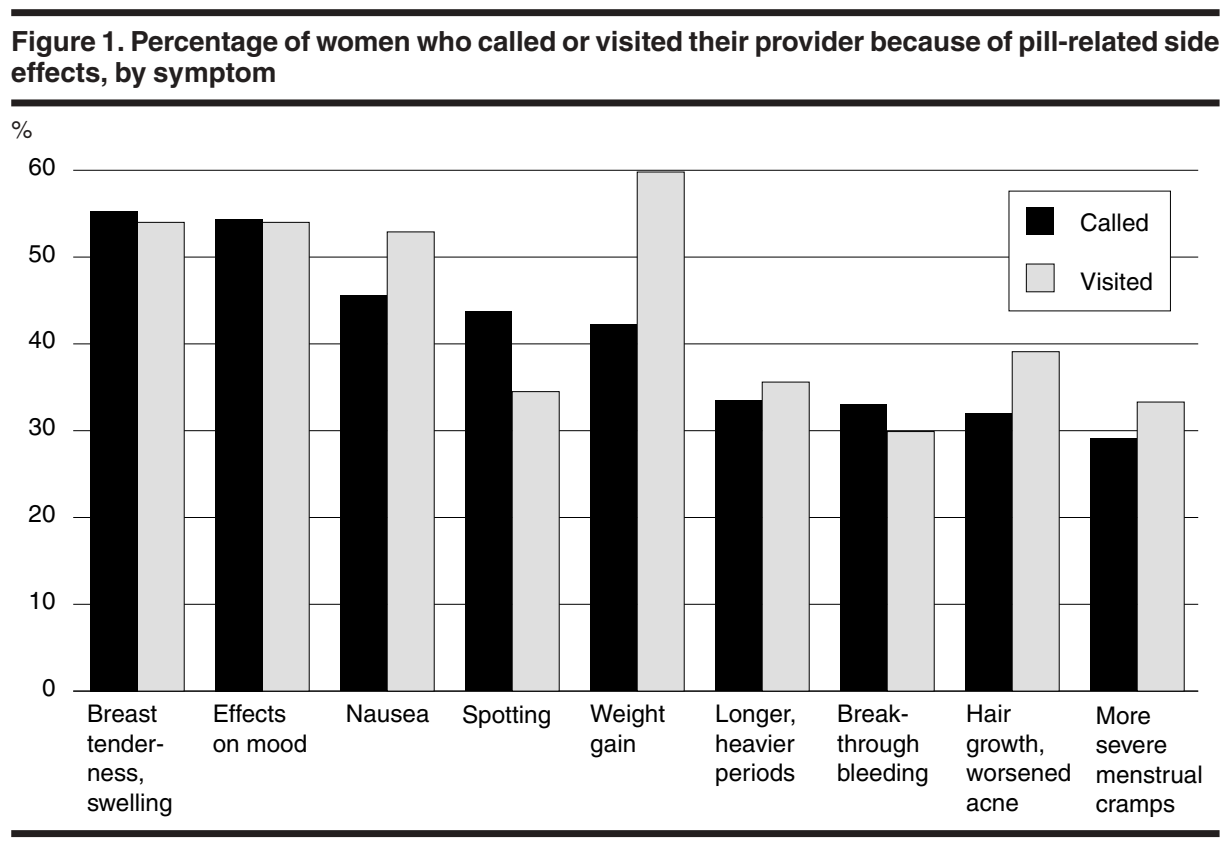

The association between higher degrees of pill satisfaction and higher ratings of patient-provider interaction is consistent with the finding from a recent study that the more thorough counseling implant users received, the lower their discontinuation rate. ${ }^{15}$ Like pill acceptability, the acceptability of the implant is highly related to the extent of bleeding irregularities, and the importance of such irregularities was lessened among study participants who had anticipated them.

Information was gathered from patients in a nonclinical, private setting, which may have made it easier for them to report compliance difficulties, early discontinuation and dissatisfaction with their method or their provider. Clinicians probably underestimate both the magnitude and the severity of such problems, given that many women discontinue taking the pill without consulting the physician who prescribed it, ${ }^{16}$ and patients probably find it difficult to express such problems in a clinical setting. The frequency of problems reported in this and other studies supports the notion that, at least from a patient's perspective, considerable room exists for improvement.

Because of the degree of detail in the questionnaire used for this study, as well as the large number of participants, we were able to use statistical analyses that permit differentiation of closely related factors. Thus, we were able to control for potential confounding and identify how various factors independently affected the outcome. Second, the prospective design minimized the possibility of recall bias that is present in retrospective or cross-sec- tional studies of contraceptive use. ${ }^{17}$

Additionally, our sample was considerably larger and more representative of the universe of oral contraceptive users than were those in earlier studies, which tended to be small and to focus on adolescents. ${ }^{18}$ While our sample was designed to be nationally representative of pill users, it is difficult to evaluate how generalizable the results are, given the paucity of recent nationally representative data on such women.

The most up-to-date data indicate that the mixture of providers from whom women receive oral contraceptives is similar to that in the present study. In the mid1980 s, an estimated $67 \%$ of women who used pills, IUDs or diaphragms relied on physicians in private practice for these services. ${ }^{19}$ In 1994, 36\% of women making a family planning visit received services from a clinic and $64 \%$ from private physicians or health maintenance organizations; Planned Parenthood sites served $30 \%$ of women receiving services from clinics. ${ }^{20}$

By comparison, $42 \%$ of women in our sample obtained services through Planned Parenthood, and 58\% went to private providers. While Planned Parenthood patients are thus overrepresented in our study population, the national data show that Planned Parenthood clients differ from family planning clinic clients in general chiefly in that they are slightly less likely to be teenagers. ${ }^{21}$

An important limitation of the study is the rate of follow-up- $64 \%$ for the twomonth questionnaire. While this rate compares favorably with those of other mail surveys, ${ }^{22}$ it nevertheless raises the possi- bility that women who enrolled in the study but failed to return the questionnaire systematically differed from women who returned it. However, we feel that an appreciable degree of such bias is unlikely, for two reasons. First, respondents were quite similar to nonrespondents with respect to characteristics recorded at enrollment. Second, we used telephone followup for all women who did not respond by mail. Responses to the key outcome measures from the group followed up by telephone were very similar to those from women who responded by mail, and both groups were similar to nonrespondents. Another limitation is that women might tend to attribute problems with the method to such factors as poor counseling rather than to their use-related behavior.

Our findings suggest that difficulties associated with the use of oral contraceptives are common, and that improving use may be a responsibility shared among pill users, manufacturers and providers. They also suggest a number of steps that may improve the situation, though additional research is required to test the effectiveness of these measures. Manufacturers should recognize that providing informative, easy-to-understand written materials may improve compliance. Some of the problems that precipitate compliance difficulties, low levels of method satisfaction, and pill-related calls and visits to providers are expected; therefore, health care providers should be the focal point of efforts to help women become more successful users. These efforts should take the form of thorough counseling and careful cultivation of the patient-provider relationship, particularly with new users or women returning to oral contraceptive use.

Specifically, providers should discuss the transient nature of most side effects, the health benefits of oral contraceptives and instructions for correct use. Furthermore, they should take the opportunity during followup contact to evaluate consistency of use (e.g., patient calls about spotting may indicate heightened chances of compliance difficulties). Finally, pill users should establish a regular pill-taking time, carefully read literature that accompanies the pill package and know what to do if pills are missed, including using a backup method when indicated. Our findings suggest that these efforts may result in increased compliance and patient satisfaction with oral contraceptives.

\section{References}

1. Rosenberg MJ, Waugh MS and Long S, Unintended pregnancies and use, misuse, and discontinuation of oral (continued on page 104) 


\section{Compliance, Counseling...} (continued from page 92)

contraceptives, Journal of Reproductive Medicine, 1995, 40(5):355-360.

2. Hannaford PC, Combined oral contraceptives: do we know all of their effects? Contraception, 1995, 51(6):325-327.

3. Hatcher RA et al., eds., Contraceptive Technology, 16th rev. ed. New York: Irvington Publishers, 1994.

4. Ibid.

5. Rosenberg MJ et al., Compliance and oral contraceptives: a review, Contraception, 1995, 52(3):137-141.

6. Trussell J et al., Contraceptive failure in the United States: an update, Studies in Family Planning, 1990, 21(1):51-54; and Jones EF and Forrest JD, Contraceptive failure rates based on the 1988 NSFG, Family Planning Perspectives, 1992, 24(1):12-19.

7. Rosenberg MJ, Waugh MS and Long S, 1995, op. cit. (see reference 1).

8. Ibid.

9. Rosenberg MJ et al., 1995, op. cit. (see reference 5).
10. Greenland S, Modeling and variable selection in epidemiologic analysis, American Journal of Public Health, 1989, 79(3):40-49.

11. Galassi JP, Schanberg R and Ware WB, The Patien Reactions Assessment: a brief measure of the quality of the patient-provider medical relationship, Psychological Assessment, 1992, 4(3):346-351.

12. DuRant R et al., Influence of psychosocial factors on adolescent compliance with oral contraceptives, Journal of Adolescent Health Care, 1984, 5(1):1-6; Jay MS, DuRant $\mathrm{RH}$ and Litt IF, Effect of peer counselors on adolescent compliance in use of oral contraceptives, Pediatrics, 1984 73(2):126-131; and Oakley D, Sereika S and Bogue EL, Oral contraceptive pill use after an initial visit to a family planning clinic, Family Planning Perspectives, 1991 23(4):150-154

13. Rosenberg MJ, Waugh MS and Meehan TE, Use and misuse of oral contraceptives: risk indicators for poor pill taking and discontinuation, Contraception, 1995 51(5):283-288.

14. Potter L et al., Measuring compliance among oral contraceptive users, Family Planning Perspectives, 1996 28(4):154-158.
15. Diaz J, Faundes A and Olmos P, Bleeding complaints during the first year of Norplant implants and their impact on removal rate, Contraception, 1996, 53(2):91-95.

16. Grady W, Hayward M and Florey F, Contraceptive discontinuation among married women in the United States, Studies in Family Planning, 1988, 19(4):227-231.

17. Rosenberg MJ, Waugh MS and Meehan TE, 1995, op. cit. (see reference 13)

18. Rosenberg MJ et al., 1995, op. cit. (see reference 5).

19. Orr MT and Forrest JD, The availability of reproductive health services from U.S. private physicians, Family Planning Perspectives, 1985, 17(2):63-69 1985.

20. Frost JJ, Family planning clinic services in the United States, 1994, Family Planning Perspectives, 1996, 28(3):92-100

21. Ibid.

22. Rimm EB et al., Effectiveness of various mailing strategies among nonrespondents in a prospective cohort study, American Journal of Epidemiology, 1990, 131(6):1068-1071. 